\title{
The Microstructure of an Aged TiAlW Alloy
}

\author{
H. Zhang, L. L. He, H. Q. Ye
}

Shenyang National Laboratory for Materials Science, Institute of Metal Research, Chinese Academy of Sciences, Shenyang 110016, P.R.China

TiAl alloys have been regarded as one of the strongest candidate materials for light and high temperature structural materials. In the Ti-Al binary phase diagram, the $\beta$ phase only appears at high temperature. By adding $\beta$ phase stabilizing elements such as $\mathrm{Cr}, \mathrm{W}, \mathrm{Nb}, \mathrm{Mo}$, ect., the primary $\beta$ phase field can be extended to lower temperature. $\beta$ phase can develop and provide attractive combinations of properties[1]. Gas atomized Ti-48.4at\%Al-1.9at\%W powder, manufactured by Crucible Compaction Metals Inc., with maximum powder size of $500 \mu \mathrm{m}$ was vacuum encapsulated in stainless steel and consolidated by hot isostatic pressing at $1250^{\circ} \mathrm{C}$ and $200 \mathrm{MPa}$ for 2 hours. The stepped cool treatment was applied. TEM observations were performed in a JEOL-2010 high-resolution transmission electron microscope.

After aging at $950^{\circ} \mathrm{C}$ for 5 hours, the $\beta$ precipitates appear as shown in Fig. 1a. The average length for long axis of $\beta$ phase is about $20 \mathrm{~nm}$. When aged at $950^{\circ} \mathrm{C}$ for 96 hours, the $\beta$ precipitates became larger apparently as shown in Fig. 1b. The average length for long axis of $\beta$ phase is about $90 \mathrm{~nm}$. The inset in Fig. $1 \mathrm{~b}$ is the corresponding selected area electron diffraction pattern (SAED). The OR between $\beta$ and $\gamma$ can be written as: $[\overline{1} 01]_{\gamma} / /[111]_{\beta},(111)_{\gamma} / /(\overline{1} 10)_{\beta}$. In fact the above OR is the well known K-S relationship between f.c.c. and b.c.c. structure except that the $\gamma$-TiAl phase is ordered.

When viewed along [111 $]_{\gamma} / /[\overline{1} 10]_{\beta}$, a typical TEM morphology of the $\beta$ precipitates is shown in Fig. 2a. There are six different needle variants, indicated by A, B, C, D, E and F. Fig. $2 b$ is the corresponding SAED. We can identify six $\beta$ variants diffractions. From these diffractions, one can certainly obtain that $\beta$ phase is b.c.c structure instead of B2 structure in the literature [1,2]. In order to understand the six different growth directions of $\beta$ phase, the OR between $\beta$ and $\gamma$ phase and the atom arrangement on close packed planes of two phases are considered. As shown in Fig. 3, on $(111)_{\gamma}$ plane there are three $60^{\circ}\langle 110\rangle_{\gamma}$ direction; on $(\overline{1} 10)_{\beta}$ plane there are two $\left.70.52^{\circ}<111\right\rangle_{\beta}$ direction. In accordance with the OR between $\gamma$-TiAl and $\beta$ phase, the two $\langle 111\rangle_{\beta}$ direction will parallel to any one among three $\langle 110\rangle_{\gamma}$ direction, then we can have six group superposition of close-packed plane of $\gamma$-TiAl and $\beta$ phase. This indicates six different variants on $(111)_{\gamma} / /(\overline{1} 10)_{\beta}[3]$.

References

[1] B. J. Inkson et al., Acta Metall. Mater., 41 (1993) 2876

[2] G. Ignacio et al., Intermetallics, 9 (2001) 373

[3] Thanks to D. Y. Seo for providing of the materials used in this study. 


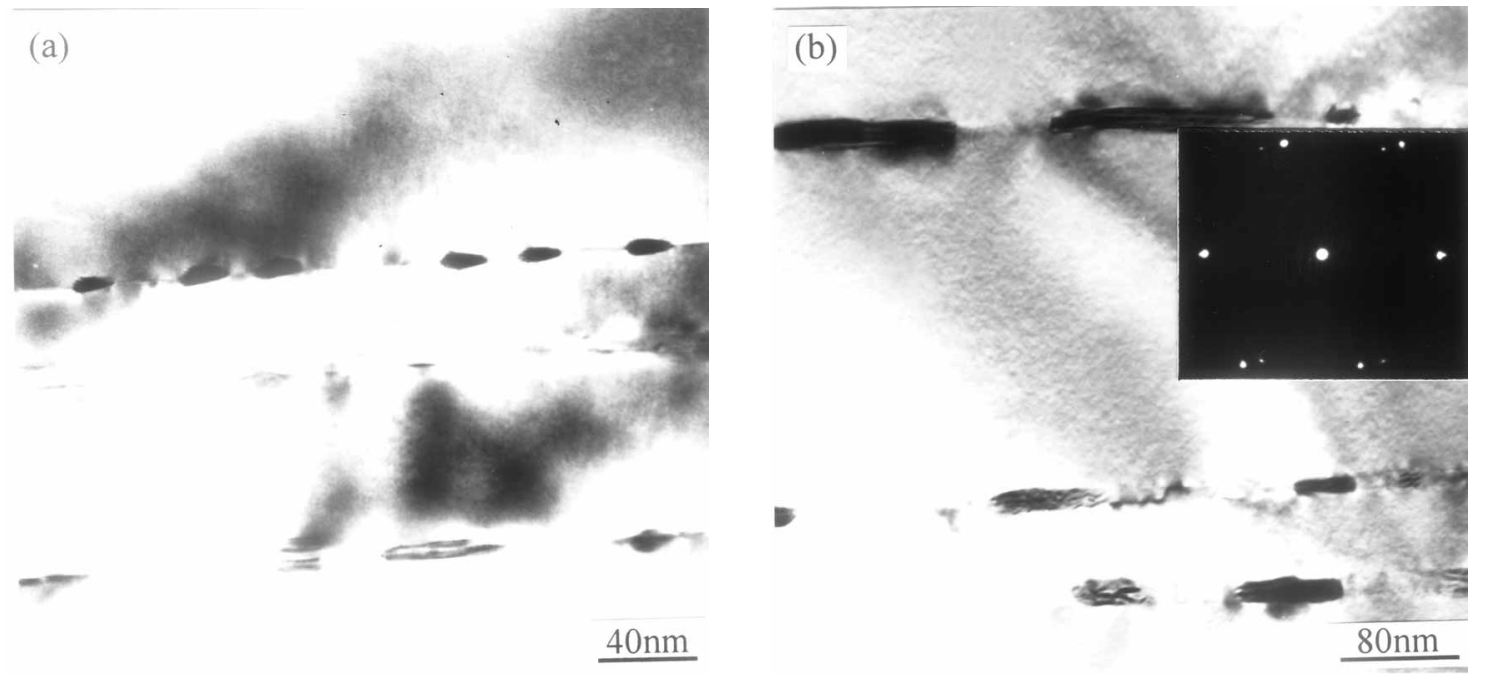

FIG. 1 The morphology of $\beta$ precipitates, viewed along $[\overline{1} 01]_{\gamma} / /[111]_{\beta}$ (a) aged at $950{ }^{\circ} \mathrm{C}$ for 5 hours, (b) aged at $950{ }^{\circ} \mathrm{C}$ for 96 hours.
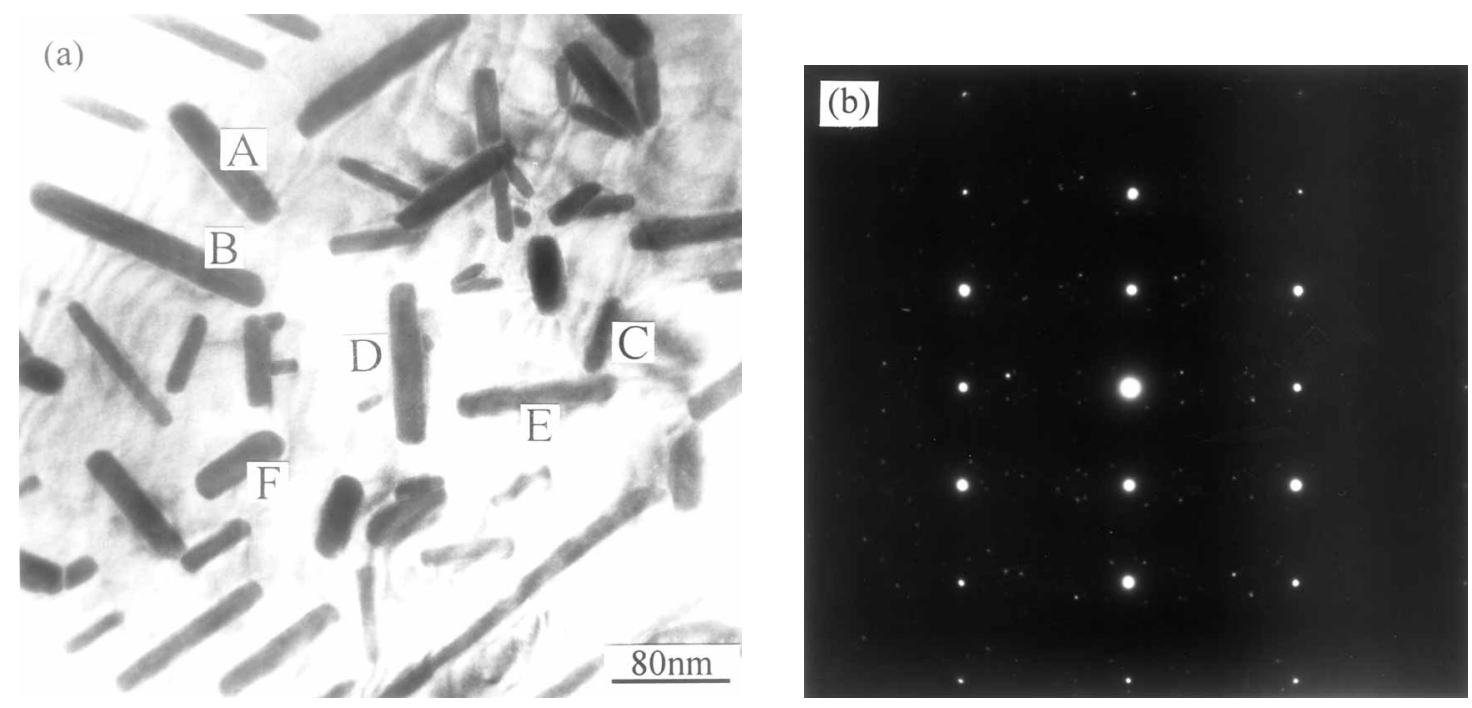

FIG. 2(a) A typical TEM image of $\beta$ precipitates, viewed along $[111]_{\gamma} / /[\overline{1} 10]_{\beta}$ (b) The corresponding SAED.
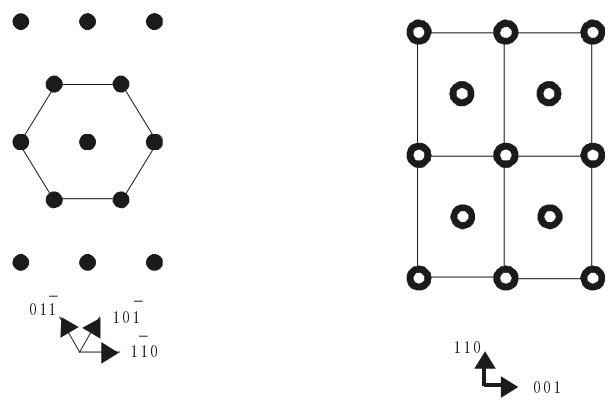

FIG. 3 Lattice points of $(111)_{\gamma}$ (full circle) and $(\overline{1} 10)_{\beta}$ (open circle) planes. 